\title{
Research on Intelligent Agriculture Monitoring System Based on Internet of Things
}

\author{
Miao Zhuang \\ Xi'an International University, Xi'an, Shaanxi, PR. China
}

\begin{abstract}
Keywords: Intelligent Agriculture, Monitoring System, Internet of Things
\end{abstract}
\begin{abstract}
Using the combination of Zig Bee technology and 3G communication technology, different agricultural bases and related equipment are combined to form a wireless sensor network system, and then the sensor parameters of different agricultural bases are collected to control the conditions of different agricultural bases more precisely and achieve remote equipment and personnel information exchange between the composition of the IOT-based intelligent agricultural monitoring system. The system has been tested many times to prove that the system has good stability, real-time monitoring, collecting information, environmental control, is widely used in farms remote management.
\end{abstract}

\section{Introduction}

Chinese agricultural production has always been relying on the traditional mode of production, wasting a lot of manpower and resources, but also caused environmental pollution and damage, is not conducive to the sustainable development of agriculture. Therefore, how to adapt to the requirements of agricultural production in the harmonious society in the new era and to design a new system to meet the requirements by using the existing technologies and platforms has become an urgent problem to be solved. Wisdom agriculture uses technologies such as internet of things, cloud storage, and Zig Bee to achieve accurate agricultural monitoring and management.

At present, Chinese agriculture is being developed from an individual mode of small-scale cultivation to an intensive and large-scale base cultivation mode [1], and various problems such as irrigation, fertilization and insect pests gradually emerge. Therefore, how to implement scientific management with high efficiency, informationization and safety for large-scale and large-scale agricultural bases is a top priority problem to be solved. At present, most of the environmental monitoring of agricultural bases in China adopts the traditional method, that is, monitoring by artificial means, which greatly increases the unnecessary labor cost and reduces the work efficiency. Internet of Things Uses communication technologies such as local networks and the Internet to connect sensors, controllers, machines, personnel and objects through new ways to form a system in which people and things, things and things are connected to achieve informationalization, remote management and control Intelligent. Based on the Internet of Things, this study proposes a smart agricultural monitoring and control system. Through this system, users can gather environmental information on large-scale agricultural bases without space constraints, and at the same time, can carry out visual and precise control over them realize the centralized and efficient management of modern agriculture.

\section{The System Overall Plan}

The monitoring system is based on the existing technology and is composed of embedded gateways, RFID, Zig Bee and various sensor modules. Based on the three-tier architecture of IoT, the intelligent monitoring system is designed and implemented based on intelligent agriculture monitoring including Android client and Web client system. Through the sensor to collect the corresponding data, and then use Zig Bee wireless technology to complete the data from the sensor to the embedded gateway transmission, and then according to the TCP protocol to enable data 
transmission from the embedded gateway to the Web server, the data analysis to form information in the Android client End and Web client display, to achieve the environment parameters in the agricultural greenhouse in the appropriate client and mobile terminal display capabilities. At the same time based on the sensor feedback information on the greenhouse control equipment for simple control to ensure the stability of environmental parameters. In addition, the system also provides Web users and mobile users with a friendly display interface, management and control interface, giving users a good experience.

\section{The On-Site Monitoring System}

Site monitoring system consists of Android phone client, embedded gateway and Zig Bee module. Android mobile phone client is mainly used to achieve human-friendly interactive interface. Into the wisdom of agricultural monitoring system interface can list a variety of environmental parameters within the greenhouse, such as greenhouse temperature and humidity information, the presence of toxic gases, whether someone broke into the greenhouse, shed light intensity.

The sensors on the four Zig Bee modules collect the environmental parameters in the greenhouse. The temperature and humidity sensors collect the temperature and humidity information in the greenhouse in real time. The broad-spectrum gas sensors detect the indoor harmful gases (CO, SO2, etc.) Whether it exceeds the standard; human detection of the main greenhouse crops play a protective role, when someone intruded into the greenhouses, it will be sensed and timely warning; sensors will collect the data sent to the Zig Bee module, and then by Zig Bee's self-organization the network is passed to the Zig Bee coordinator in the entire network. In addition, the fan based on the CC2530 plays an exhaust effect. The coordinator sends the data to the embedded gateway through the RS 232 serial port for corresponding processing. The embedded gateway processes the received data transmitted by the Zig Bee coordinator and transmits the data to the mobile client via the local area network. The embedded gateway processes the data sent by the mobile client and controls the corresponding sensors, growth lights and fans. On-site monitoring subsystem needs to meet the real-time data storage analysis, data acquisition, network connectivity and other functions.

As a low-rate, low-complexity, low-loss and low-cost wireless network technology, Zig Bee technology has gradually become the first choice for short-range communication applications. From the requirement of agricultural greenhouse, the data type that general shed needs to transmit do not require high communication speed, so using Zig Bee method instead of traditional wiring is very feasible. Taking into account the general agricultural base has a shorter control distance, measuring points and more equipment and other characteristics, the use of Zig Bee's Mesh networking. Mesh network by the router, coordinating node, a number of terminal nodes, belonging to multi-hop network system. In the network nodes can communicate directly, each communication by one or more routing nodes relay, and finally passed to the destination node.

Adopt ARM-Linux controller mode, the part of hardware chooses ARM Cortex-A9 series as the microprocessor of the embedded controller, this series processor has the characteristics of high performance, strong processing power and low power consumption; the software part adopts Linux operation System, which has the advantages of multi-tasking, multi-user, high compatibility, simple interface operation, support for multiple platforms and good security.

Video surveillance using PTZ HD network camera, it can not only manually control the camera rotation, but also through the Web or mobile App to control, with a standard H.264 algorithm, and can support CIF, D1 two resolutions for wireless networks ; Support 360-degree camera rotation; Wi-Fi, Bluetooth can transmit data for inconvenient wiring occasions. The camera will capture the video data encoded by the built-in encoder and wireless network transmission to the management center, at the same time the decoder will decode the received data to play the video.

\section{The Embedded Operating Platform Design}

ARM - Linux controller mode [3]. The hardware part selects the ARM11 series core as the 
microprocessor of the embedded controller, which has the characteristics of high processing power, high performance and low power consumption. The software part of the operating system adopts the Linux operating system, which has the advantages of high compatibility, Multi-user, multi-tasking, interface is simple, good security, support for multiple platforms and so on. Embedded system structure shown in Figure 4, the dotted line part of the embedded system software architecture and built-in applications. Embedded operating platform based on Linux operating system to build embedded WEB server to meet the remote management center browser information exchange. To meet the large amount of data such as video information processing, the system introduces lightweight SQLite database, easy to store and manage information, the use of common CGI technology WEB server and database connection. Through the connection between the web page and the database, the query request of the user can be converted into the query command of the database, and the query result can be returned to the remote user through the web page. The remote user can send the control instruction through the browser and send the command to the Zig Bee The coordinator connects to the serial port. After the Zig Bee coordinator receives the information from the serial port, it sends the information to the corresponding Zig Bee terminal node to control the device. ARM - Linux controller parameters acquisition and control of equipment flow: first is to initialize the network, and then regularly collect the relevant data stored in SQLite database, the collection interval determined by the staff, and finally on the WEB dynamically update the data. Under normal circumstances, the system defaults to automatic control mode, the system uses a more robust fuzzy PID algorithm [4] to more human-based control of the base: combined with sensor-related data collected, the controller by fuzzy reasoning analysis According to the analysis, the control signal is sent to the related equipment to regulate the crop growth environment so as to make the environmental conditions of the base reach the optimum condition for crop growth. Upon receiving the control signal from the remote management center, the system immediately switches to manual mode. Embedded system control flow shown in Figure 5, first send Zig Bee network initialization command; initialisation is completed, the system regularly collect sensor feedback data and synchronously updated to the database, at the same time, real-time reflected in the WEB page; operator observation To update the data in real time, to determine whether the manual operation.

\section{The Wireless Network Subsystem}

Wireless network subsystem using China Unicom 3G (3rd generation mobile communication technology) network [5] seamlessly connected with the Ethernet to achieve the user's remote monitoring. With the maturity of $3 G$ technology and wide coverage of the network, the high anti-interference ability, high compatibility and high data transmission rate of 3G network make it fully meet the transmission needs of various data in the monitoring process of agricultural base. The Ethernet adopts the special line connection mode, and the remote management center applies for a fixed public IP address from the network service provider. The on-site monitoring subsystem receives the related information, and the embedded control platform sends the information to the 3G network through 3G-DTU (3G data unit) sent out, through the base station and network server equipment to achieve seamless $3 G$ network and Ethernet connectivity; IP network services provided by the fixed IP transmission to the remote management center to achieve on-site and long-distance management center connection. On the contrary, the management center of the relevant control information can also be released to the on-site monitoring subsystem through the line, on-site monitoring subsystem receives the control information, through a series of reverse processing of information to achieve the ultimate remote control.

\section{Conclusion}

The system collects the corresponding data through each sensor, uses Zig Bee wireless technology to transmit the data from the sensor to the embedded gateway, and then transfers the data from the embedded gateway to the Web server according to the TCP protocol, and then analyzes the formed 
information Display on Android client and Web client. The system can realize the real-time display of environment parameters (including the corresponding mobile terminal display) in the agricultural greenhouses. At the same time, the control devices in the greenhouses can be simply operated according to the feedback information of the sensors so as to ensure the stability of environmental parameters.

\section{Acknowledgement}

Research project of Shaanxi Provincial Education Department Technical Scheme and Algorithm Design of Intelligent Water Saving Irrigation System under Mountain Environment (18JK21**)

\section{References}

[1] Li Zhen, Zeng Jiangyuan, Chen Quan, Bi Haiyun. Measurement and Model Construction of Vegetation Complex Dielectric Constant [J] .China Science: Geosciences. 2014 (03)

[2] Deng Xiuxin. Modern Agriculture and Agricultural Development [J]. Huazhong Agricultural University (Social Science Edition). 2014 (01)

[3] Qian Zhihong, Wang Yijun. Overview of Wireless Sensor Networks for Internet of Things [J] .Journal of Electronics \& Information Technology 2013 (01)

[4] Ding Zhenfan. Serial communication application programming based on Java [J]. Microcomputer and Applications. 2012 (13)

[5] LI Huiyun, HE Zhenwei, LI Li, LU Gang. Research on HTML5 Technology and Application Patterns [J] .Telecommunications Science. 2012 (05) 